\title{
ASSOCIATION OF X-RAY FLARES WITH SOLAR \\ CORONAL ACTIVE REGIONS
}

\author{
P. R. SENG UPTA \\ Institute of Applied Manpower Research New Delhi-1, India
}

\begin{abstract}
Summary. It is known that the flare component of solar X-ray emission is sensitive to the level of solar activity. The location of the X-ray flaring region is not directly known but can be inferred from the location of the associated $\mathrm{H} \alpha$ flare. A detailed study of more than 4000 solar X-ray flares recorded by UI and the NRL detectors during past eight years has shown that $85 \%$ of these flares definitely occurred in the active regions. For the rest $15 \%$ no definite conclusion was possible because either $\mathrm{H} \alpha$ flare data was not available or no $\mathrm{H} \alpha$ flare was reported within \pm 15 min of these X-ray flares.
\end{abstract}

An analysis of soft X-ray flares associated with three active regions, viz. McMath Regions 10607, 10618 and 11128, which occurred in February-March, 1970 and January, 1971, has led to the following conclusions:

(i) X-ray flares generally occur in the active regions.

(ii) The frequency of occurrence, intensity and the spectral hardness of the soft X-ray flare emission are related to solar activity level and are consistent with the index of activity derived by Sengupta $(1971,1974)$.

(iii) Flare X-ray emission increases with the activity level and is maximum during the final phase of the growth. Flare emission declines markedly during the decay phase of the activity.

(iv) Eruptive flares and hard X-ray bursts generally occur during the final phase of the growth.

\section{References}

Sengupta, P. R.: 1971, 'A Study of Solar X-ray Emission and its Effect on the Earth's Ionosphere',

D.Sc. Thesis, Department of Electronics, I.I.T., Kharagpur, India.

Sengupta, P. R.: 1974, Space Res. 14, 461. 